\title{
Patrioten für drei Wochen. Nationale Identifikation und die Fußballeuropameisterschaft 2012
}

\author{
Michael Mutz
}

Zusammenfassung: Bei Welt- und Europameisterschaften im Fußball sympathisieren die Zuschauer in der Regel mit dem Nationalteam ihres Landes. Die Nationalmannschaft wird zum Ankerpunkt für nationale Identifikation und - bei sportlichem Erfolg - zur Quelle von Nationalstolz. Nationale Identifikation kann sich als patriotische Verbundenheit ausdrücken, aber auch in einen negativen, übersteigerten Nationalismus umschlagen, der die eigene Nation idealisiert und Fremdgruppen abwertet. Der Beitrag untersucht patriotische und nationalistische Einstellungen vor, während und nach der Fußball-EM 2012. Dazu werden Längsschnittdaten aus einer studentischen Stichprobe ausgewertet. Die Ergebnisse zeigen, dass das Anschauen der Spiele der deutschen Mannschaft mit einem starken Anstieg von Patriotismus und einem moderaten Zuwachs von Nationalismus verbunden ist. Diese Zuwächse resultieren aus dem Erleben starker, positiver Emotionen, der Einbindung in kollektive Rituale und der Aneignung nationaler Symbole während der EM. Wenige Tage nach dem Ende der EM fielen diese Effekte aber schon erkennbar schwächer aus. Eine nachhaltige Veränderung patriotischer oder nationalistischer Einstellungen dürfte die EM deshalb nicht bewirkt haben.

Schlüsselwörter: Kollektive Identität $\cdot$ Patriotismus $\cdot$ Nationalismus $\cdot$ Nationalstolz $\cdot$ Fußball

\section{Patriots for three weeks. National identification and the European Football Championship 2012}

\begin{abstract}
During a FIFA World Cup or a European Football Championship, spectators usually sympathise with their own country's national team. The national team becomes a significant point of reference for national identification and - in the event of athletic success - a source of national pride. National identification, however, may be expressed as a patriotic feeling of belonging, but may also turn into negative, exaggerated nationalism which idealises the own nation and devalues outsiders. This paper investigates the patriotic and nationalistic attitudes before, during and after the European Football Championship 2012. To this end, longitudinal data from a student sample are analysed. Findings show that viewing the games of the German team is associated with a steep increase in patriotism and a moderate increase in nationalism. This rise results from the
\end{abstract}

(C) Die Autor(en) 2013. Dieser Artikel ist auf Springerlink.com mit Open Access verfügbar

M. Mutz $(\bowtie)$

Institut für Sportwissenschaften, Georg-August-Universität Göttingen,

Sprangerweg 2, 37075 Göttingen, Deutschland

E-Mail: michael.mutz@sport.uni-goettingen.de 
experience of strong, positive emotions, the involvement in collective rituals and the adoption of national symbols during the European Cup. In contrast, these effects were already markedly weaker only a few days after the end of the tournament. Thus, the European Football Championship has probably not produced a lasting change in patriotic or nationalistic attitudes.

Keywords: Collective identity $\cdot$ Patriotism $\cdot$ Nationalism $\cdot$ National pride $\cdot$ Football

\section{Patriotes pour trois semaines. L'identification nationale pendant le championnat d'Europe de football de 2012}

Résumé: Pendant les championnats du monde et d'Europe de football les spectateurs sympathisent généralement avec l'équipe de leur pays. L'équipe nationale devient ainsi le point de fixation de l'identification nationale et, en cas de victoire, source de fierté nationale. L'identification nationale peut se manifester sous forme de sentiment patriotique mais aussi virer au nationalisme négatif exacerbé basé sur l'idéalisation de la nation d'appartenance et la dépréciation de groupes étrangers. Cet article étudie les attitudes patriotiques et nationalistes avant, pendant et après le championnat d'Europe de football de 2012. Pour ce faire, des données longitudinales portant sur un échantillon d'étudiants ont été analysées. Les résultats montrent que le fait de regarder les matches de l'équipe allemande est corrélé avec une forte poussée de patriotisme et une progression modérée du nationalisme. Ces progressions s'expliquent par l'expérience de fortes émotions positives, la participation à des rituels collectifs et l'appropriation des symboles nationaux pendant la durée de l'Euro. Cependant, quelques jours après la fin de l'Euro, ces effets s'étaient déjà sensiblement atténués. Aussi l'Euro ne devrait-il pas avoir provoqué de changement durable des attitudes patriotiques ou nationalistes.

Mots-clés: Identité collective $\cdot$ Patriotisme $\cdot$ Nationalisme $\cdot$ Fierté nationale $\cdot$ Football

\section{Einleitung}

Fußballweltmeisterschaften und Europameisterschaften gehören $\mathrm{zu}$ den sportlichen Mega-Events, die ein globales Publikum erreichen. Die Mannschaften, die bei diesen Turnieren antreten und den Sieger untereinander ausspielen, repräsentieren Nationalstaaten. Insofern sind Welt- und Europameisterschaften zwar globale Sportereignisse, sie sind zugleich aber eingerahmt in eine nationale Symbolik, die sich unter anderem am Abspielen der Nationalhymnen vor den Spielen und der Präsenz von Nationalflaggen im Stadion manifestiert. Die meisten Zuschauer, ob im Stadion oder vor dem Fernseher, identifizieren sich insbesondere mit der Nationalmannschaft ihres eigenen Landes und fiebern mit dieser mit. Siege des eigenen Nationalteams erzeugen bei der weit überwiegenden Mehrheit der Zuschauer Freude und Stolz, während Niederlagen mit Enttäuschung und Trauer verbunden sind. Die Nationalteams verkörpern die Nation und werden für die Dauer einer EM oder WM zu einem herausragenden Ankerpunkt nationaler Zugehörigkeit und Verbundenheit.

In Deutschland ist diese Rolle der Fußballnationalmannschaft für die Erzeugung nationaler Identität insbesondere seit der Weltmeisterschaft 2006 in den Fokus öffentlicher und gesellschaftspolitischer Debatten gerückt. Zur WM im eigenen Land präsentierten sich „die Deutschen“ nicht nur als gute Organisatoren und überwiegend freundliche, kosmo- 
politische Gastgeber, sondern auch als Patrioten, die ihre Verbundenheit mit der eigenen Nation unerwartet offen zeigten und durch Flaggen am Auto oder am Balkon, Accessoires wie Schals, Perücken und Ketten oder als schwarz-rot-goldene Gesichtsbemalung offen zum Ausdruck brachten. Dieser überraschend „entspannte“, „fröhliche“ und „unverkrampfte“ Umgang mit nationalen Symbolen wurde seit der WM 2006 zu jedem großen Fußballturnier in den Feuilletons ausgiebig kommentiert und diskutiert. Die Kommentare reichten dabei von der ablehnenden Sorge vor einem neuen Nationalismus in Deutschland bis zu zahlreichen Stimmen, die eine „Normalisierung“ im Selbstverständnis der Deutschen als längst überfällig begrüßten (zur Debatte vgl. Dembowski 2009; Hebeker und Hildmann 2007; Schediwy 2012).

Jenseits feuilletonistischer Debatten ergeben sich auch für die Sozialwissenschaft mehrere relevante Forschungsfragen: 1) Erstens ist grundsätzlich zu fragen, ob Nationalstolz tatsächlich durch ein Fußball-Großereignis messbar ansteigt. 2) Zweitens ist zu prüfen, ob es sich bei diesem Nationalstolz um einen harmlosen Patriotismus handelt, bei dem das Feiern und der Sport im Vordergrund stehen, oder ob auch nationalistische Einstellungen zunehmen, in denen sich eine Überhöhung der deutschen Nation und die Abwertung anderer Nationen ausdrücken. 3) Drittens ist zu erklären, wie genau nationale Identifikation während eines Fußballturniers erzeugt und aktualisiert wird. 4) Schließlich stellt sich eine vierte Frage: Ist der vermutete größere Nationalstolz während eines Fußball-Events kurz danach wieder verflogen oder handelt es sich um einen nachhaltigen Effekt von längerer Dauer? Damit sind vier Forschungsfragen umrissen, die der vorliegende Beitrag ein Stück weit erschließen will.

\section{Kollektive Identität und der Nationalstaat}

Menschen fühlen sich zu unterschiedlichen sozialen Kollektiven zugehörig, die ihre kollektive Identität prägen. Eine Person könnte sich beispielsweise als Berliner, Christ, Akademiker oder Sozialdemokrat beschreiben. Der Referenzpunkt kollektiver Identität ist in jeder dieser Beschreibungen eine größere soziale Gruppe, zu der man dazugehört und mit der man sich verbunden fühlt. Zugleich wird damit eine Abgrenzung nach außen vollzogen, zu allen Nicht-Berlinern, Nicht-Christen oder Nicht-Akademikern. Kollektive Identität basiert auf drei Merkmalen: a) dem Wissen, zu einem sozialen Kollektiv dazuzugehören, b) dem Gefühl der Verbundenheit zu diesem Kollektiv und c) der damit immer (implizit) verbundenen Grenzziehung zwischen „uns“ und „den anderen“ (vgl. Ashmore et al. 2004; Eisenstadt und Giesen 1995; Turner et al. 1987). ${ }^{1}$ In vielen Situationen bestimmen diese kollektiven Zugehörigkeiten das Denken und Handeln von Personen. Sowohl in der Sozialpsychologie als auch in der Soziologie und Politikwissenschaft befassen sich deshalb zahllose Beiträge mit den Formen, Ursachen und Folgen kollekti-

1 Dabei wird nicht vorausgesetzt, dass man die anderen Gruppenmitglieder persönlich kennt. Es genügt bereits, dass man sich als zusammengehörig wahrnimmt. Vor allem in sozialpsychologischen Experimenten wurde dies immer wieder belegt: „members of a social group seem often to share no more than a collective perception of their own social unity and yet this seems to be sufficient for them to act as a group" (Turner 1982, S. 15). 
ver Identität (vgl. z. B. Brewer 1991; Brubaker und Cooper 2000; Calhoun 1994; Fuchs et al. 1993; Jenkins 1996; Kaina 2009; Sen 2007; Tajfel 1982; Tajfel und Turner 1986; Wieviorka 2003).

Nationalstaaten sind einer der zentralen Referenzpunkte für die kollektive Identität einer Person. Menschen sind beispielsweise bereit, über Steuern und Sozialabgaben Kollektivgüter zu finanzieren oder den Nationalstaat im Rahmen von Wehrdiensten vor Angriffen zu schützen. Sie bringen anderen Angehörigen des Nationalstaates in größerem Ausmaß Vertrauen und Solidarität entgegen (vgl. Delhey 2004). Dies alles setzt voraus, dass sich Menschen mit der eigenen Nation identifizieren. Eine solche Identifikation ist nun nicht „,naturgegeben“, sondern weitestgehend sozial konstruiert: Nationalstaaten erzeugen und fördern diese Identifikation durch nationale Symbole wie Flaggen, Hymnen, Uniformen, Nationaldenkmäler, Gedenktage oder Wappen, durch die Betonung einer nationalen Geschichte und Kultur, gemeinsamen Werten und Traditionen und nicht zuletzt einer gemeinsamen Sprache (vgl. Anderson 1983; Hobsbawm 1991; Hroch 2005). Obwohl sich die Menschen eines Nationalstaates nicht persönlich kennen, glauben sie daran, eine zusammengehörige Gemeinschaft zu sein. Benedict Anderson (1983) hat Nationalstaaten deshalb als, ,imagined communities“" bezeichnet.

Nationale Identität wird zwar auch, wie es das Konzept der Staatsnation beschreibt, durch die Verfassung gefördert, die den Staatsbürgern Freiheits- und Grundrechte einräumt und so unter den Bürgern formale Gleichheit herstellt (vgl. Lepsius 1982). Oft wird aber argumentiert, dass Freiheitsrechte und abstrakte Formen der Solidarität wie Steuern und Sozialabgaben allein nicht genügen würden, um dauerhaft eine belastbare kollektive Identität innerhalb eines Nationalstaates zu erzeugen und aufrechtzuerhalten (vgl. Calhoun 2005; Depenheuer 1995). Vielmehr seien Vergemeinschaftungsprozesse ohne emotionale Bindungen an die Gruppe schwer vorstellbar. Insofern ist auch eine nationale Identität auf emotional aufgeladene Ereignisse angewiesen, durch die der Einzelne in symbolischer Form an der Nation teilhaben kann und wodurch die Nation als Gemeinschaft unmittelbar erlebt wird. Solche Ereignisse, die ein Verbundenheitsgefühl hervorrufen, waren in der Vergangenheit häufig militärische Bedrohungen oder Naturkatastrophen, die die Bürger zusammenrücken ließen und deren erfolgreiche Bewältigung im Nachhinein als Grundlage für nationalen Stolz diente. Auch Gedenktage und die damit verbundenen Festakte können diese Funktion erfüllen, wenn etwa zum Tag der Deutschen Einheit an die friedliche Revolution in der DDR und die Wiedervereinigung als ,historische Leistungen“ erinnert wird. Herausragende Sportereignisse, ob das „Wunder von Bern“ oder das „Sommermärchen“, finden ebenfalls ihren Platz im kollektiven Gedächtnis der Nation und können emotionale Bindungen an die Nation erzeugen.

Vor allem Sozialpsychologen haben immer wieder betont, dass Individuen nach einer positiven kollektiven Identität streben (vgl. Luhtanen und Crocker 1992; Tajfel und Turner 1986; Turner 1982). Sie wollen sich zu einer Gruppe dazugehörig fühlen, die möglichst hohe Anerkennung genießt, einen hohen Status besitzt, über viele Ressourcen verfügt und die sie als erfolgreich wahrnehmen können. Eine positive kollektive Identität resultiert vor allem aus dem Vergleich mit Fremdgruppen: Schneidet die Eigengruppe im Vergleich zur Fremdgruppe besser ab, stärkt das die Identifikation mit der eigenen Gruppe. Wendet man dies auf die Zugehörigkeit zu einer Nation an, scheint es ganz verschiedene Dimensionen zu geben, auf denen man sich mit anderen Ländern vergleichen kann: Als Deut- 
scher könnte man etwa den wirtschaftlichen Wohlstand, den umfangreichen Sozialstaat, die kulturellen Leistungen der deutschen „Dichter und Denker“ oder aber Erfolge im Spitzensport positiv bewerten und auf dieser Grundlage eine Bindung an die Nation aufbauen. Ireneusz Pawel Karolewski und Andrzej Marcin Suszycki (2011, S.45) sprechen hier vom „self-esteem booster effect“: Die Identifikation mit der als erfolgreich wahrgenommenen Nation wird zur Ressource für das individuelle Selbstwertgefühl.

Eine starke Identifikation mit der Nation wird nun allerdings keineswegs als ausschließlich positives Merkmal von Personen betrachtet. Die normativen Debatten kreisen vielmehr um die Frage, an welchem Punkt die Identifikation mit dem Nationalstaat in unerwünschten Nationalismus umschlägt. In diesem Zusammenhang wird oft zwischen wünschenswertem „Patriotismus“ und einem schädlichen „Nationalismus“ differenziert (vgl. Fleiß et al. 2009; Kosterman und Feshbach 1989; Weiss und Reinprecht 1998): Während ersterer die emotionale Verbundenheit mit dem eigenen Land meint, ohne dass dies mit der Abwertung von Fremden verbunden ist, bezeichnet letzterer die Idealisierung und Überbewertung der eigenen Nation, bei der alle anderen Nationen zugleich als weniger wertvoll betrachtet werden. Wenn sich an die Verbundenheit mit der eigenen Gruppe die Abwertung ,der anderen“ anknüpft, schlägt nationale Identifikation um in Nationalismus. Solche nationalistischen Einstellungen beschreibt Daniel J. Levinson (1950, S. 107) wie folgt: ,blind attachment to certain national cultural values, uncritical conformity with the prevailing group ways, and rejection of other nations as out-groups“. ${ }^{2}$ Für Theodor W. Adorno und Kollegen hängt Nationalismus eng mit faschistischen, antisemitischen und ethnozentristischen Einstellungen zusammen (Adorno et al. 1950). Richard Rorty (1998, S. 3) geht ebenfalls davon aus, dass nationale Identifikation nur in einem gewissen Ausmaß erwünscht sein kann, da der überzogene Glaube an die Überlegenheit der eigenen Nation Kriegslust und Imperialismus begünstige: „National pride is to countries what self-respect is to individuals: [...] Too much national pride can lead to bellicosity and imperialism, just as excessive self-respect can produce arrogance“.

Nationale Identifikation wird also einerseits als Grundlage für Solidarität und Zusammenhalt innerhalb eines Nationalstaates angesehen, andererseits in der gesteigerten Form des Nationalismus als Grundlage für Faschismus und Imperialismus verhandelt. Sport im Allgemeinen und Fußball im Besonderen können nun Quellen für Nationalstolz sein, der als Patriotismus oder als Nationalismus zum Ausdruck kommen kann.

\section{Fußball als Ankerpunkt für nationale Identifikation}

Ein wichtiger Ankerpunkt für das Erleben nationalstaatlicher Zugehörigkeit sind ohne Zweifel sportliche Mega-Events wie Olympische Spiele oder Welt- und Europameisterschaften im Fußball (vgl. van Hilvoorde et al. 2010; Houlihan 1997; Schrag 2009). Im Fokus der Endrunden zu den Welt- und Europameisterschaften im Fußball stehen Mannschaften, die einzelne Nationalstaaten repräsentieren. Das Zuschauerinteresse richtet

2 Allerdings spricht Levinson (1950) dabei nicht explizit von Nationalismus, sondern von „Pseudo-Patriotismus“. Was er damit allerdings meint, wäre nach heutiger Terminologie mit dem Begriff Nationalismus zu bezeichnen. 
sich dabei in besonderer Weise auf die Mannschaft aus dem eigenen Land. Sportliche Wettkämpfe, bei denen Nationalteams gegeneinander antreten, erlauben soziale Vergleiche zwischen Eigengruppe und Fremdgruppe auf besonders einfache und direkte Art und Weise. Schneiden die Sportlerinnen und Sportler des eigenen Landes nun besonders erfolgreich ab, ist dies eine wichtige Quelle für Nationalstolz und positive Identifikation mit dem eigenen Land. Jede Medaille und jeder Sieg, sei es bei den Olympischen Spielen oder einem internationalen Fußballturnier, werden so zu einem anschaulichen Exempel für die Leistungsfähigkeit, Stärke, ja Überlegenheit der eigenen Nation und dürften die Bindung an die Nation und die Selbstkategorisierung als Angehöriger dieser Nation entsprechend verstärken.

Aber auch unabhängig vom sportlichen Erfolg in Form von Siegen und Medaillen gewinnt die nationale Zugehörigkeit während eines sportlichen Großereignisses ungemein an Salienz, denn nahezu die gesamte Medienberichterstattung wird über nationale Kategorien strukturiert: Mit Blick auf die TV-Berichterstattung zu den Olympischen Spielen schreibt Gunter Gebauer, dass die Medien nicht mehr vollständig einem bestimmten Wettbewerb folgen, sondern nach Belieben von einem Wettkampf zum nächsten springen, sobald irgendwo ein deutscher Sportler an den Start geht. Die Berichterstattung erhält ihre dramaturgische Einheit nicht länger durch den sportlichen Wettkampf, sondern die Übertragung wird an den deutschen Farben entlang inszeniert: „Alle Zuschauer sind in Bewegung: auf der Spur der Deutschen. [...] Das neue Darstellungsprinzip, die neue Einheit ist der deutsche Nationalismus" (Gebauer 1996, S. 264). Auf diese Weise wird der Sport zum Generator von nationaler Identifikation stilisiert und zur Bühne erkoren, auf der „deutsche Tugenden“ - Fleiß, Entschlossenheit, Stärke, Disziplin - von erfolgreichen deutschen Sportlerinnen und Sportlern verkörpert werden sollen. Und der Nationalstolz des mitfiebernden Fernsehpublikums dürfte dabei umso stärker steigen, je mehr Siege „wir“ erringen. Der Sport kann nun, gerade wenn er medial als Symbol für die Stärke und den Erfolg der Nation in Szene gesetzt wird, leicht nationalistische Einstellungen begünstigen. Hierin liegt, wie Gebauer (ebd., S. 269) anmerkt, eine Gefahr: „Aus der Identifizierung mit ,unseren' Sportlern werden Unterschiede gewonnen gegen alle diejenigen, die nicht, wir' sind. Das neue Darstellungsprinzip des Fernsehens folgt den Deutschen durch die Räume und Zeiten hindurch, meidet die anderen Sportler und macht sie zu Fremden, die nicht weiter interessieren“. Was Gebauer für die Olympischen Spiele beobachtet, gilt indes auch für die mediale Inszenierung der Fußball-WM und -EM.

Im Fußball dürfte die Assoziation der Nationalmannschaft mit der Nation und die Grenzziehung zwischen ,uns“ und „den Anderen“ sogar noch ausgeprägter sein als bei den Olympischen Spielen: Zum einen stehen bei den Olympischen Spielen in den meisten Sportarten individuelle Athletinnen und Athleten im Fokus. Bei Mannschaftssportarten wie Fußball rückt hingegen das Team in den Vordergrund. In diesen Fällen wird die sportliche Leistung von einem Kollektiv erbracht, und es wird leichter, Siege und Erfolge mit der Nation zu assoziieren. ${ }^{3}$ Zum anderen steigt die Identifikation mit der Fußball-

3 Ob bei der Biathlon-Staffel, beim Ruder-Achter oder eben im Fußball: Oft heißt es auch in der medialen Berichterstattung einfach: „Deutschland ist Weltmeister“ oder „Wir holen den Titel“. In Individualsportarten ist dieser Sprachgebrauch unüblich, weil offensichtlich ist, dass Individuen die sportliche Leistung vollbringen. 
nationalmannschaft simpel daher, weil Fußball eine herausgehobene Stellung im deutschen Sportsystem innehat. Fußball ist die populärste Sportart in Deutschland, und ein WM- oder EM-Titel im Fußball dürfte dementsprechend an „Wert“ kaum zu überbieten sein. Während bei den Olympischen Spielen die erfolgreichen deutschen Dressurreiter, Fechter oder Kanuten nur für wenige Tage mediale Aufmerksamkeit erhalten und einem breiteren Publikum ohnehin nicht bekannt sind, stehen die deutschen Fußballnationalspieler ständig im medialen Fokus und genießen eine öffentliche Aufmerksamkeit, die der von Pop-Stars kaum nachsteht. Bei wichtigen Spielen der deutschen Mannschaft versammelt sich nahezu das gesamte Land vor den Fernsehschirmen - zur EM 2012 waren es pro Spiel etwa 25 Mio. Zuschauer. Das allein unterstreicht den potenziellen Einfluss, den die DFB-Elf auf den Nationalstolz der Deutschen ausüben kann.

Wie genau sollen die Fußballspiele der Nationalmannschaft nun aber die Identifikation der Zuschauer mit dem Nationalstaat beeinflussen? Eine erste Voraussetzung erscheint geradezu als selbstverständlich: sportlicher Erfolg. Damit man sich mit einem Kollektiv identifiziert, muss dieses Kollektiv über positive Eigenschaften verfügen. Würde die DFB-Auswahl ihre Spiele durchweg verlieren, einen unattraktiven Fußball spielen oder aus unsympathischen Spielern bestehen, wäre sie kein attraktiver Ankerpunkt für kollektive Zugehörigkeit und auch keine Quelle für Nationalstolz. Darüber hinaus haben einige Arbeiten im Detail beschrieben, durch welche weiteren Mechanismen kollektive Identifikation im Sport erzeugt wird (vgl. Cottingham 2012; Ismer 2011; Schediwy 2012): Kollektive Emotionen, gemeinsame Rituale und die Präsenz von Gruppensymbolen werden dabei als zentrale Elemente angesehen.

Fußball besitzt ein ungemein großes Potenzial, Emotionen bei den Zuschauern auszulösen. Diese Emotionen können im sportlichen Geschehen ihren Ursprung haben, sie können aber auch durch kollektive Rituale erzeugt werden. Fan-Gesänge, Sprechchöre, rhythmisches Klatschen und Springen etc. sind Aktivitäten, die die Aufmerksamkeit der Beteiligten in die gleiche Richtung lenken und oft bereits Gemeinschaftsgefühle und Emotionen auslösen, auch wenn das Spiel noch gar nicht läuft. Gerade wenn Emotionen im Kollektiv erlebt werden und in kollektive Rituale eingebunden sind, steigert das ihre Intensität. Die eigene Freude, Nervosität, Trauer oder Euphorie finden dann einen Widerhall bei den anderen Beteiligten: Man steckt sich gegenseitig an, und die Stimmung schaukelt sich wechselseitig hoch (vgl. Riedl 2008; Schäfer 2010; von Scheve et al. 2012). Diesen außeralltäglichen, quasi rauschhaften Zustand kollektiver Erregung und Ekstase hat Emile Durkheim (1981, S.283 ff.) als Ursprung religiöser Empfindungen beschrieben. Ähnlich wie bei religiösen Ritualen wird in solchen Momenten der alltägliche Bewusstseinszustand transzendiert: Die Beteiligten empfinden sich nicht länger als individuelle Akteure, sondern sie gehen voll in der Gruppe auf. Kollektive Rituale und Emotionen führen dazu, dass sich die Beteiligten für eine gewisse Zeitdauer unmittelbar als zusammengehörige Gemeinschaft erleben und sich mit der Gruppe identisch fühlen.

Bei Spielen der Fußball-Nationalmannschaft ist diese Gruppe, mit der man sich verbunden fühlt, die Nation. Diese Gruppenbindung wird durch nationale Symbole erzeugt, die bei Spielen der Fußball-Nationalmannschaft allgegenwärtig sind. Ob im Stadion oder beim Public Viewing: die Zuschauer sind umgeben von schwarz-rot-goldenen Farben, Flaggen oder Emblemen. Diese nationalen Symbole werden im Zuge des Fußballspiels mit Emotionen aufgeladen, sodass sich ein starkes, positives Gefühl an diese anhaftet, 
das, wie man vermuten kann, auch nach dem Schlusspfiff noch bestehen bleibt. ${ }^{4}$ Die meisten Zuschauer sind nun, zumindest wenn sie ein EM- oder WM-Spiel außerhalb der eigenen vier Wände ansehen, nicht nur passive Rezipienten dieser nationalen Symbole, sondern sie eignen sich diese Symbole aktiv an, indem sie sich selbst in den schwarzrot-goldenen Farben kleiden oder schminken, ihre Autos und Balkone mit DeutschlandFähnchen dekorieren oder die Nationalhymne vor dem Spiel mitsingen. Fußballspiele bieten insofern eine Bühne, auf der die eigene Identifikation mit der Nation expressiv gezeigt, inszeniert und emotional erlebt werden kann und auf der man sich zugleich als Teil einer weitaus größeren nationalen (Fußball-)Gemeinschaft erleben kann (vgl. dazu auch Schediwy 2012).

Wenngleich das Anschauen von Fußballspielen an öffentlichen Plätzen, in Kneipen und Bars seit 2006 in Deutschland Hochkonjunktur hat, sehen die meisten Zuschauer die EM- und WM-Spiele nach wie vor als Fernsehzuschauer, also weder beim Public Viewing noch im Stadion. Doch auch die TV-Berichterstattung hat sich in den letzten Jahren stark verändert und ist inzwischen, gerade beim Fußball, darauf ausgerichtet, Emotionen zu transportieren und nationale Verbundenheitsgefühle zu verstärken. Sven Ismer (2011) hat das im Vergleich der Weltmeisterschaften von 1974 und 2006 herausgearbeitet: Zur WM 1974 herrschte ein Klima kritischer Distanz zur Nationalmannschaft und zu nationalen Symbolen generell, die Medienberichterstattung war von einem sachlichen, nüchternen Ton geprägt, die Leidenschaft der Kommentatoren hielt sich in Grenzen. Im Kontrast dazu zielten die Fernsehberichte 2006 viel stärker darauf ab, Emotionen einzufangen und zu transportieren: u. a. durch Live-Bilder aus den Fan-Zonen und Party-Meilen vor dem Spiel; durch Vorberichte, die Spannung aufbauen und Sympathie mit der deutschen Mannschaft erzeugen sollen; durch einen stärker emotionalen Stil des Kommentierens während der Spiele. Durch diese gewandelte Form der TV-Berichterstattung dürften auch beim Fernsehzuschauer intensivere Emotionen ausgelöst und eine größere Identifikation mit der Nation erzeugt werden.

Dass der Nationalstolz in der deutschen Bevölkerung in der Tat durch herausragende Fußballereignisse beeinflusst wird, zeigen Analysen der Allgemeinen Bevölkerungsumfrage der Sozialwissenschaften aus dem Jahr 2006 (vgl. Ahlheim und Heger 2008, S. 46). Während der Fußball-WM 2006 stieg der Nationalstolz der Deutschen von WM-Spiel zu WM-Spiel an und erreichte vor dem Halbfinale gegen Italien den Höhepunkt (vgl. Abb.1). Nach dem verlorenen Halbfinale knickte der Anteil derjenigen, die stolz auf Deutschland waren, deutlich ab, blieb aber noch einige Wochen auf höherem Niveau. Im August, etwa 4 Wochen nach dem ,Sommermärchen“, pendelte sich der Nationalstolz allerdings wieder auf dem Niveau aus der Zeit vor der Fußball-WM ein. Wenngleich die dargestellten Werte auf Daten beruhen, die im Querschnitt erhoben wurden, und die Fallzahlen zu den einzelnen Befragungszeitpunkten zudem recht klein sind, spiegeln die Befunde doch die euphorische Stimmung über die Leistung der DFB-Auswahl gut wider.

4 Emile Durkheim hat allgemeiner beschrieben, wie sich Gefühle auf „Dinge“ übertragen, die dadurch zu Symbolen werden. Erst durch diese Übertragung auf Symbole können die erlebten Gefühle überdauern, denn dann werden sie durch die Präsenz dieser Symbole und Zeichen wachgehalten. Das Symbol wird dann ,geliebt, gefürchtet und geachtet; ihm ist man dankbar; ihm opfert man sich“(Durkheim 1981, S. 302). 


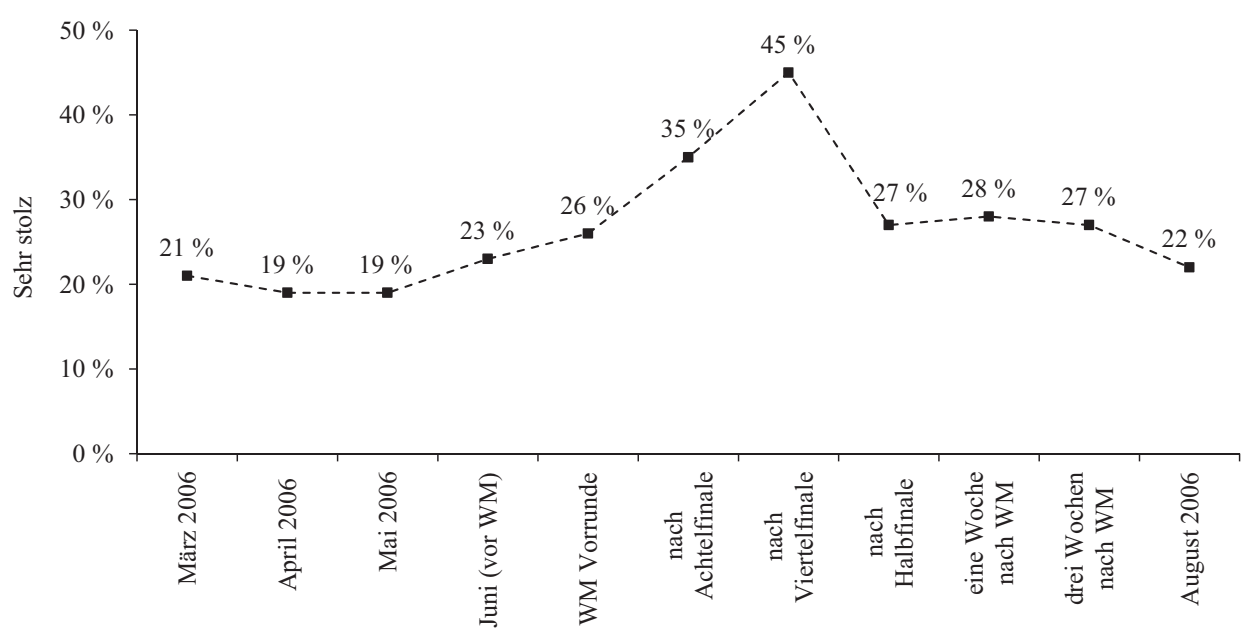

Abb. 1: Nationalstolz der Deutschen im Verlauf der Fußball-WM 2006. (Quelle: Ahlheim und Heger 2008, S. 46, sowie eigene Berechnungen am ALLBUS 2006. Die Abbildung zeigt den Anteil der Befragten zu jedem Zeitpunkt, die ,sehr stolz“ sind, Deutsche(r) zu sein.)

Der Nationalstolz der Deutschen befand sich während der WM 2006 ohne Zweifel für kurze Zeit im Ausnahmezustand. ${ }^{5}$

Warum aber ebbte der Nationalstolz bereits wenige Wochen nach der WM wieder ab? Matthias Junge argumentiert vor der Folie postmoderner Gegenwartsdiagnosen, dass feste, dauerhafte, unhinterfragte Referenzpunkte für Zugehörigkeit in der „flüchtigen Moderne" nicht länger zur Verfügung stehen und stattdessen durch episodenhafte, expressive und emotionale Formen von Sozialität ersetzt werden. Public Viewing ist für ihn geradezu ein Paradebeispiel für solche posttraditionalen Gemeinschaftsformen, nach denen postmoderne Menschen eine „unstillbare Sehnsucht“ entwickeln: Das efferveszente, rauschhafte Erleben von Gemeinschaft gestatte jeweils für einen Augenblick „die kollektive Erinnerung an eine einstmals unproblematische Einheit von Individuum und Gesellschaft“ (Junge 2008, S. 194). Diese kollektive Euphorie lässt sich indes nicht auf Dauer stellen: Nach dem Ende des Fußball-Spektakels verliert die nationale Zugehörigkeit rasch ihre Salienz, und die auf die Nation bezogenen Gemeinschaftsgefühle verschwinden. Der Nationalstaat tritt dann wieder in den Hintergrund und dient selbstreflexiven Individuen lediglich als einer von vielen möglichen und insofern kontingenten Ankerpunkten für kollektive Identifikation.

5 Korrelationen zwischen der Rezeption von herausragenden Sportereignissen und der Empfindung von Nationalstolz können auch für die Niederlande belegt werden (vgl. van Hilvoorde et al. 2010): Jene Befragten, die die Fußball-EM 2008 oder die Olympischen Spiele 2008 im Fernsehen verfolgt haben, waren besonders stolz darauf, Niederländer zu sein. Ihr Nationalstolz und ihr Gefühl der nationalen Zugehörigkeit lagen deutlich über den Werten jener Personen, die sich die beiden Sportereignisse nicht angesehen haben. 
Aus den bisherigen Überlegungen ergeben sich vier Forschungsfragen, die für die empirische Studie leitend waren. Da in der empirischen Untersuchung zwischen Patriotismus und Nationalismus unterschieden wird, kann jede dieser Forschungsfragen zum einen im Hinblick auf patriotische als auch auf nationalistische Einstellungen formuliert werden:

- Das Ansehen der Spiele der deutschen Mannschaft bei der UEFA EURO 2012 dürfte mit der Zunahme von Patriotismus bzw. von Nationalismus einhergehen. Zumindest bis zum Halbfinale ist dies zu erwarten, da das DFB-Team alle Vorrundenspiele sowie das Viertelfinale gewinnen konnte und erst im Halbfinale aus dem Turnier ausschied.

- Wurden die EM-Spiele an einem öffentlichen Ort angesehen, ist ein besonders starker Anstieg von Patriotismus bzw. von Nationalismus zu vermuten, weil anzunehmen ist, dass beim Public Viewing die Erlebnisintensität - auch im Hinblick auf das Erleben nationaler Zugehörigkeit - größer ist als vor dem Fernseher zu Hause.

- Der vermutete Anstieg von nationaler Identifikation dürfte sich ursächlich aus dem Erleben von kollektiven Emotionen und der (oft in Rituale eingebundenen) Aneignung nationaler Symbole beim Fußball ergeben. Für beide Erklärungsfaktoren müssten folglich positive Effekte auf Patriotismus bzw. Nationalismus zu erkennen sein.

- Schließlich dürfte der Einfluss der Fußball-EM auf Patriotismus bzw. Nationalismus nicht von Dauer sein. Das Ansehen der EM-Spiele sollte nur während der EM, nicht aber nach der EM die Identifikation mit der Nation beeinflussen.

\section{Zur Methodik}

\subsection{Stichprobe und Datenerhebung}

Um die Frage zu erschließen, ob sich im Verlauf eines sportlichen Mega-Events wie der UEFA EURO 2012 die Identifikation mit der eigenen Nation verändert, ist es wünschenswert, Längsschnittdaten zu analysieren, also die gleichen Personen mehrmals zu befragen. Für die vorliegende Studie wurden die Einstellungen zum Nationalstaat zu drei Zeitpunkten, nämlich vor, während und nach der EM 2012 erhoben. Repräsentative Längsschnitterhebungen sind bekanntlich mit besonders großem Aufwand und sehr hohen Kosten verbunden und waren im Rahmen der hier vorgestellten Untersuchung nicht zu realisieren. Geht es allerdings vor allem darum, eine relativ neue und bislang weitestgehend unbeantwortete Forschungsfrage in einem ersten Schritt zu explorieren, erscheint es legitim, dafür eine nicht-repräsentative Stichprobe zu verwenden. Welche Einschränkungen damit für die Generalisierbarkeit der Befunde einhergehen, wird am Schluss noch genauer diskutiert.

Für die folgende Analyse wurden deshalb Studierende in sportwissenschaftlichen Bachelor-Studiengängen an drei deutschen Universitäten befragt: der Georg-August-Universität Göttingen, der Technischen Universität Dortmund und der Humboldt-Universität zu Berlin. An jedem Standort wurde jeweils eine Lehrveranstaltung aus dem Sommersemester 2012 ausgewählt. Zu drei Zeitpunkten im Semester wurden diese Studierenden gebeten, einen kurzen Fragebogen auszufüllen. Die erste Erhebung fand am 8./9. Mai 
2012 statt, genau einen Monat vor dem Beginn der Fußball-EM (t1). Die zweite Erhebung wurde am 26./27. Juni 2012 durchgeführt, nachdem die DFB-Auswahl ihre drei Vorrundenspiele sowie ihr Viertelfinale gewonnen hatte und in das Halbfinale eingezogen war (t2). Eine dritte Erhebung fand nach der EM statt, am 10./11. Juli 2012, etwa zwei Wochen nachdem das deutsche Team im EM-Halbfinale gegen Italien ausgeschieden war (t3). Am Ende des Fragebogens wurde von den Studierenden ein selbst generierter, persönlicher Code abgefragt, der ihnen zum einen Anonymität gewährte, zum anderen aber das spätere Zuordnen der Fragebögen für die Längsschnittanalyse ermöglichte. Für 80 Studierende konnten die Fragebögen auf Basis des persönlichen Codes eindeutig zugeordnet werden, sodass die Einstellungsveränderungen über die drei Messzeitpunkte im Längsschnitt analysiert werden konnten.

\subsection{Indikatoren für Patriotismus und Nationalismus}

Innerhalb der bereits erläuterten Bandbreite nationaler Identifikation müssen die Konzepte Patriotismus und Nationalismus theoretisch gegeneinander abgegrenzt und mit geeigneten Items und Skalen operationalisiert werden. Für die Studierendenbefragung zur Fußball-EM wurden zu jedem der drei Erhebungszeitpunkte die Einstellungen zur Nation mit Hilfe von Items erfasst, die in ähnlicher Form Teil der Wiener Nationalismus- und Patriotismus-Skala waren und sich zur Messung von nationaler Identifikation bewährt haben (vgl. Weiss et al. 2009a, 2009b; Weiss und Reinprecht 1998). ${ }^{6}$

1. Patriotismus meint eine positive, affektive Bindung an die eigene Nation bzw. eine „Liebe zum eigenen Land“, die nicht mit der Exklusion und Abwertung von Fremdgruppen einhergeht (Weiss et al. 2009a). Patriotische Einstellungen wurden mit drei Items erfasst, die auf eben diese emotionale Verbundenheit zur Nation rekurrieren (vgl. Tab. 1). Die Antwortvorgaben reichten von „stimme gar nicht zu“ bis „stimme voll zu“. Die Skala, die nachfolgend als „Patriotismus“ bezeichnet wird, wurde auf einen Wertebereich von 1 bis 7 transformiert. Höhere Werte stehen für stärker ausgeprägte patriotische Einstellungen.

2. Nationalismus bezeichnet eine übersteigerte, bedingungslose und kritiklose Bindung an die eigene Nation, die mit der Abwertung anderer Nationen und der Ausgrenzung von Fremden einhergeht (Weiss et al. 2009b). Zur Messung nationalistischer Einstellungen werden vier Items herangezogen, in denen sich die unbedingte Verehrung der Nation und der Glaube an die Überlegenheit der eigenen Nation ausdrücken (vgl. Tab. 1). Die Befragten konnten auch hier ihre Zustimmung von ,stimme gar nicht zu“ bis „stimme voll zu“ abstufen. Die aus diesen Items gebildete Skala „Nationalismus“ wurde ebenfalls auf einen Wertebereich von 1 bis 7 transformiert, wobei größere Werte einen höheren Nationalismus ausdrücken.

6 Zum Teil wurden die Formulierungen der für Österreich entwickelten Items für Deutschland leicht angepasst. 
Tab. 1: Skalen zur Messung von Nationalismus und Patriotismus

\begin{tabular}{lll}
\hline Skala & „Patriotismus“ & „Nationalismus“ \\
\hline Items & Deutscher zu sein, empfinde ich als & Die Welt wäre besser, wenn die \\
& wichtigen Teil meiner Identität. & Menschen in anderen Ländern so \\
& Ich liebe Deutschland. & wären wie die Deutschen. \\
& Wenn ich die deutsche Fahne sehe, & Es ist die unbedingte Pflicht jedes \\
fühle ich mich großartig. & Deutschen, die nationale Geschichte \\
& & und Kultur zu ehren. \\
& & Jeder Deutsche sollte Deutschland \\
& & unterstützen, selbst wenn das Land \\
& & im Unrecht ist. \\
& & Aufgrund der bedeutenden \\
& & geschichtlichen Erfahrungen sollte \\
& & Deutschland viel mehr Einfluss in der \\
& & Welt haben. \\
Konsistenz & $\alpha=0,68$ & $\alpha=0,65$ \\
Wertebereich & $1-7$ & $1-7$ \\
Mittelwert & $\mathrm{M}=3,52$ & $\mathrm{M}=2,46$ \\
\hline
\end{tabular}

\subsection{Erklärungsvariablen}

Als Erklärungsvariablen wurden zum zweiten Messzeitpunkt vier weitere Angaben erhoben, von denen vermutet wurde, dass sich mit ihrer Hilfe eine Veränderung patriotischer und nationalistischer Einstellungen erklären lässt. Erfragt wurde,

- welche der insgesamt vier EM-Spiele (Vorrundenspiele und Viertelfinale) der deutschen Nationalmannschaft sich die Befragten angesehen haben;

- welche der vier EM-Spiele der deutschen Nationalmannschaft sie an einem öffentlichen Ort, also beim Public Viewing angesehen haben;

- inwieweit die Befragten bei den Spielen der deutschen Mannschaft positive Emotionen erlebt haben, sich a) ,ausgelassen gefreut“ und b) ,,in jeder Minute mitgefiebert“ haben ${ }^{7}$;

- welche der folgenden Dinge die Befragten während der Europameisterschaft getan haben: a) Tragen von DFB-Fanartikeln, b) Aufhängen der deutsche Flagge, c) Mitsingen der Nationalhymne, d) Anziehen oder Schminken in schwarz-rot-goldenen Farben, e) Skandieren von Schlachtrufen oder Mitsingen bei Fan-Gesängen. ${ }^{8}$

7 Die Befragten konnten ihre Zustimmung zu den Aussagen (1) „Ich habe mich ausgelassen gefreut" und (2) „Ich habe in jeder Minute mitgefiebert" auf einer vierstufigen Rating-Skala abstufen. Die beiden Angaben wurden zu einem Index (Wertebereich 0-3) zusammengefasst, wobei größere Werte stärkere Emotionen ausdrücken.

8 Für jede der fünf Handlungen wurde erhoben, ob die Befragten diese Handlung während der EM ausgeführt haben. Die positiven Antworten wurden zusammengefasst und wie folgt recodiert: $(0)=$ keine Handlung, (1) = eine Handlung, (2) = zwei oder drei Handlungen, (3) = vier oder fünf Handlungen. 
Für alle vier Erklärungsvariablen ist zu vermuten, dass sich positive Zusammenhänge mit patriotischen und nationalistischen Einstellungen ergeben können. Je mehr Spiele der deutschen Mannschaft eine Person überhaupt angesehen hat und je mehr Spiele beim Public Viewing angesehen wurden, umso stärker sollten Patriotismus und Nationalismus während der Fußball-EM ansteigen. Dieser Anstieg sollte über zwei Mechanismen geschehen: Zum einen steigt die Identifikation mit der Nation durch das Erleben positiver Emotionen beim Zuschauen, zum anderen steigt sie durch die Aneignung nationaler Symbole (Flaggen, Nationalfarben) und die Partizipation an kollektiven Ritualen (Gesänge, Schlachtrufe). Man kann daher folgende kausale Beziehung vermuten: Durch das Ansehen der EM-Spiele der deutschen Mannschaft erleben Personen positive Emotionen, und sie werden in kollektive Rituale eingebunden, durch die sie sich nationale Symbole aneignen. Dadurch wird ihre Verbundenheit zur Nation verstärkt, was in einer stärkeren Bekundung von patriotischen und/oder nationalistischen Einstellungen zum Ausdruck kommen sollte.

\section{Ergebnisse der Analysen}

\subsection{Patriotismus während der Fußball-EM 2012}

In einem ersten Schritt wird geprüft, ob das Patriotismus-Niveau mit dem Anschauen der EM-Spiele der deutschen Fußballnationalmannschaft zusammenhängt. In den folgenden Analysen dient die Skala für Patriotismus während der Fußball-EM (t2) als abhängige Variable. Die vor der EM gemessenen Patriotismus-Werte (t1) fungieren als Kontrollvariable. Nach Aufnahme dieser Kontrollvariablen (im Modell I) wird nicht mehr das Niveau an Patriotismus während der EM erklärt, sondern die Veränderung im Vergleich zum Zeitpunkt t1. Die weiteren Regressionsmodelle (Modell II-IV) werden schrittweise spezifiziert, um die Kausalstruktur zu prüfen, die zwischen den unabhängigen Variablen unterstellt wird (vgl. Tab.2). Zuerst wird geprüft, ob das Ansehen der EM-Spiele der deutschen Mannschaft mit einer Zunahme der Patriotismus-Werte einhergeht. Anschließend wird untersucht, ob mit dem Ansehen der Spiele im Rahmen von Public Viewing ein

Tab. 2: Patriotismus während der Fußball-EM 2012

\begin{tabular}{lllll}
\hline & \multicolumn{5}{l}{ Patriotismus während der EM (t2) } \\
\cline { 2 - 6 } & I & II & III & IV \\
\hline Patriotismus vor der EM (t1) & $0,67^{*}$ & $0,62^{*}$ & $0,61^{*}$ & $0,53^{*}$ \\
angesehene Spiele (DFB-Auswahl) & - & $0,37^{*}$ & $0,29^{*}$ & 0,13 \\
Public Viewing (Spiele DFB-Auswahl) & - & - & $0,11^{+}$ & $-0,01$ \\
Erleben positiver Emotionen & - & - & - & $0,29^{*}$ \\
Aneignung nationaler Symbole & - & - & - & $0,24^{*}$ \\
Modellgüte $\left(\mathrm{R}^{2}\right)$ & 0,45 & 0,49 & 0,51 & 0,58 \\
\hline
\end{tabular}

Studierendenbefragung. Lineare Regressionsanalysen. Angegeben sind unstandardisierte Regressionskoeffizienten (b)

${ }^{*} p<0,05 ;{ }^{+} p<0,10$ (einseitiger Signifikanztest) 
besonders starker Anstieg verbunden ist. Schließlich prüft das Modell in einem letzten Schritt, ob die vermuteten Mediatoreffekte zu erkennen sind: Beeinflussen die EM-Spiele deshalb die patriotischen Einstellungen, weil beim Ansehen der Spiele positive Emotionen erlebt und nationale Symbole angeeignet werden? Ein solcher Mediatoreffekt wäre dann belegt, wenn die neu aufgenommenen Merkmale (positive Emotionen, Aneignung nationaler Symbole) signifikant positiv mit der abhängigen Variablen korrelieren und zugleich der Einfluss, der für das Ansehen der EM-Spiele ausgewiesen wird, insignifikant ausfällt.

(1) Im Modell I wird zunächst eine Regressionsanalyse berechnet, in der die patriotischen Einstellungen während der EM (t2) auf die patriotischen Einstellungen einen Monat vor der EM (t1) zurückgeführt werden. Erwartungsgemäß ist dieser Zusammenhang sehr stark ausgeprägt $\left(\mathrm{R}^{2}=0,45\right)$. Befragte, die schon vor der EM ein hohes Patriotismus-Niveau aufwiesen, stimmten den entsprechenden Aussagen auch während der EM stärker zu. Bei vielen Befragten haben sich die patriotischen Einstellungen im Verlauf der sechs Wochen allerdings auch verändert, denn der Zusammenhang, den Modell I ausweist, ist keineswegs perfekt. Diese Veränderung gilt es nun, in den folgenden Modellen systematisch zu erklären.

(2) Modell II prüft, ob sich die Veränderungen im Patriotismus auf das Ansehen der EM-Spiele zurückführen lassen. Zum zweiten Messzeitpunkt hätten die Befragten vier Begegnungen der DFB-Auswahl ansehen können. Die Antworten variieren hier also zwischen null (= kein EM-Spiel angesehen) und vier (= vier EM-Spiele angesehen). Wie zu erkennen ist, steigt der Erwartungswert auf der sechsstufigen Patriotismus-Skala mit jedem angesehenen Spiel der deutschen Mannschaft um 0,37 Skalenpunkte an. Wenn man annimmt, dass der Patriotismus-Wert eines Befragten, der keines der vier EM-Spiele gesehen hat, zwischen $\mathrm{t} 1$ und $\mathrm{t} 2$ konstant bleibt, dann würde man Modell II zufolge für eine Person, die alle vier Spiele gesehen hat, einen Anstieg des Patriotismus um 1,48 Skalenpunkte erwarten. Dieser Anstieg ist recht stark, und der Zusammenhang fällt entsprechend signifikant aus.

(3) Steigt das Patriotismus-Niveau dann besonders stark an, wenn die EM-Spiele an einem öffentlichen Ort, also beim Public Viewing angesehen werden? Zusätzlich zur Anzahl der angesehenen EM-Spiele der deutschen Auswahl wird in Modell III auch die Anzahl der Spiele berücksichtigt, die an einem öffentlichen Ort angesehen wurden. Wie zu erkennen ist, ist diese Differenzierung nicht nebensächlich: Bei Befragten, die die Spiele der deutschen Mannschaft beim Public Viewing gesehen haben, steigen die patriotischen Einstellungen besonders stark an. Geht man wieder davon aus, dass sich der Patriotismus-Wert eines Befragten, der kein EM-Spiel des DFB-Teams gesehen hat, zwischen $\mathrm{t} 1$ und $\mathrm{t} 2$ nicht verändert hat, dann lässt sich auf Basis von Modell III schätzen, dass bei einer Person, die alle vier Spiele im privaten Rahmen gesehen hat, der Patriotismus-Wert um 1,16 Skalenpunkte ansteigt. Hat ein Befragter hingegen alle vier EM-Spiele an einem öffentlichen Ort gesehen, müssen nochmals 0,44 Punkte hinzuaddiert werden - man würde also einen Anstieg der patriotischen Einstellungen um insgesamt 1,60 Skalenpunkte schätzen.

(4) In einem letzten Analyseschritt (Modell IV) werden nun zwei Erklärungsvariablen berücksichtigt, die spezifizieren sollen, wie die Veränderung der patriotischen Einstellungen zustande kommen. Zum einen ist das ein Indikator für die positiven Emotionen und 
die positive Erregung, die beim Anschauen der EM-Spiele erlebt wurden. Zum anderen wurde erfragt, ob sich die Befragten bei der EM nationale Symbole angeeignet haben und in kollektive Rituale eingebunden waren, z. B. die Nationalflagge aufgehängt haben, bei der Nationalhymne mitgesungen oder Schlachtrufe skandiert haben. Es ist nun anzunehmen, dass kein signifikanter Effekt für das Ansehen der EM-Spiele und die Beteiligung am Public Viewing mehr zu erkennen ist, sobald das Emotionserleben und die Aneignung nationaler Symbole während der EM in das Modell aufgenommen werden.

Die Effekte im Modell IV entsprechen exakt den Erwartungen: Die positiven Effekte für die angesehenen EM-Spiele und für das Public Viewing aus Modell III sind nun deutlich schwächer und nicht mehr signifikant. Der Effekt für Public Viewing liegt sogar fast exakt bei null. Da zugleich die beiden neu berücksichtigten Indikatoren signifikante positive Effekte anzeigen, bestätigt sich die theoretisch angenommene Kausalstruktur: Patriotische Einstellungen nehmen beim Anschauen der EM-Spiele deshalb zu, weil beim Zuschauen kollektive Emotionen erlebt und nationale Symbole angeeignet werden. Beide unabhängigen Variablen umfassen einen Wertebereich von null bis drei, und die ausgewiesenen Effekte $(0,29$ bzw. 0,24) sind von ähnlicher Stärke: Nimmt man an, dass sich das Patriotismus-Niveau von $\mathrm{t} 1 \mathrm{zu} \mathrm{t} 2$ bei jenen Befragten, die keine positiven Emotionen angegeben haben, nicht verändert hat, so würde man für die Personen, die von sehr starker Freude und Erregung bei den Spielen berichten, einen Anstieg von 0,87 Skalenpunkten erwarten. Ähnlich lässt sich der Einfluss für die Aneignung nationaler Symbole verdeutlichen: Geht man davon aus, dass der Patriotismus-Wert bei den Befragten konstant bleibt, die keine der abgefragten Handlungen ausgeführt haben, dann lässt sich für die Befragten, die mehr als drei entsprechende Handlungen angegeben haben, eine Zunahme im Patriotismus von 0,72 Skalenpunkten schätzen.

Die Modellgüte $\left(\mathrm{R}^{2}\right)$ im Modell IV liegt bei 0,58. Dieser hohe Wert ist jedoch vornehmlich darauf zurückzuführen, dass der Patriotismus-Wert vor der EM (t1) als Kontrollvariable berücksichtigt werden muss. Wie gut die Veränderung des Patriotismus während der Fußball-EM erklärt werden kann, lässt sich erst aus der Differenz der Modellgüte zwischen Modell I und Modell IV erkennen: Der Zuwachs im $\mathrm{R}^{2}$ von 0,13 verdeutlicht, dass sich der Patriotismus der Befragten während der Fußball-EM zu 13\% systematisch mit der EM - Anschauen der Spiele, Emotionen, Aneignung nationaler Symbole - erklären lässt.

\subsection{Nationalismus während der Fußball-EM 2012}

Steigt auch das Nationalismus-Niveau während der Fußball-EM (t2) an? Die folgenden Analysen sind genau so aufgebaut wie die eben besprochenen Regressionsmodelle zum Patriotismus. Erst erfolgt die Kontrolle für die vor der EM gemessenen nationalistischen Einstellungen der Befragten. Anschließend werden die interessierenden Erklärungsvariablen schrittweise in das Modell eingeführt und auf ihren Erklärungsbeitrag getestet (vgl. Tab. 3).

(1) Zunächst werden die Nationalismus-Werte der Befragten vier Wochen vor der EM (t1) als Kontrollvariable berücksichtigt (Modell I). Wie zu erwarten war, gibt es auch hier einen starken Zusammenhang zwischen den zwei Messzeitpunkten $\left(\mathrm{R}^{2}=0,32\right)$. Befragte, die vor der EM höhere Nationalismus-Werte aufwiesen, stimmten auch während der EM 
Tab. 3: Nationalismus während der Fußball-EM 2012

\begin{tabular}{lllll}
\hline & \multicolumn{5}{l}{ Nationalismus während der EM (t2) } \\
\cline { 2 - 6 } & I & II & III & IV \\
\hline Nationalismus vor der EM (t1) & $0,50^{*}$ & $0,47^{*}$ & $0,46^{*}$ & $0,48^{*}$ \\
angesehene Spiele (DFB-Auswahl) & - & $0,22^{*}$ & 0,17 & 0,03 \\
Public Viewing (Spiele DFB-Auswahl) & - & - & 0,07 & $-0,02$ \\
Erleben positiver Emotionen & - & - & - & $0,19^{+}$ \\
Aneignung nationaler Symbole & - & - & - & $0,18^{*}$ \\
Modellgüte $\left(\mathrm{R}^{2}\right)$ & 0,32 & 0,34 & 0,35 & 0,42 \\
\hline
\end{tabular}

Studierendenbefragung. Lineare Regressionsanalysen. Angegeben sind unstandardisierte Regressionskoeffizienten (b)

${ }^{*} p<0,05 ;{ }^{+} p<0,10$ (einseitiger Signifikanztest)

eher den nationalistischen Aussagen zu. Die interessierende Frage ist nun aber, ob Veränderungen zwischen $\mathrm{t} 1$ und $\mathrm{t} 2$ systematisch mit der Fußball-EM zusammenhängen.

(2) Die Befunde im Modell II lassen erkennen, dass sich das Ansehen der EM-Spiele auch auf die Befürwortung von Nationalismus positiv auswirkt. Der Effekt ist zwar deutlich schwächer als in den Analysen zum Patriotismus. Dennoch ließe sich für einen Befragten, der die vier Begegnungen der DFB-Auswahl angesehen hat, ein Anstieg im Nationalismus um 0,88 Skalenpunkte schätzen, wenn man zugleich davon ausgeht, dass die Nationalismus-Werte konstant bleiben, wenn eine Person kein Spiel der deutschen Mannschaft gesehen hat.

(3) Im nächsten Analyseschritt (Modell III) wird danach differenziert, ob die EMSpiele der deutschen Auswahl an einem öffentlichen Ort oder in einer privaten Wohnung angesehen wurden. Diese zusätzliche Unterscheidung führt aber zu keiner signifikanten Verbesserung der Erklärungsgüte des Modells: Die nationalistischen Aussagen werden von den Befragten, die am Public Viewing teilgenommen haben, nur geringfügig stärker befürwortet als von jenen Befragten, die die EM-Spiele zu Hause angesehen haben. Dieser Unterschied deutet zwar in die erwartete Richtung, ist aber äußerst gering und statistisch nicht signifikant.

(4) Schließlich werden im Modell IV zum einen die Selbsteinschätzung der erlebten positiven Emotionen und zum anderen die Angaben über die Aneignung nationaler Symbole einbezogen. Geht man auch hier davon aus, dass nationalistische Einstellungen deshalb beim Ansehen der EM-Spiele hervorgerufen und verstärkt werden, weil die Rezipienten Emotionen erleben, in Rituale einbezogen werden und sich nationale Symbole aneignen, so müsste im Modell IV kein signifikanter Effekt für das Ansehen der EM-Spiele und die Beteiligung am Public Viewing mehr zu erkennen sein. Stattdessen müssten signifikante und positive Effekte für die beiden neu aufgenommenen Merkmale ausgewiesen werden.

Die Befunde stützen diese Annahme: Das Ansehen der EM-Spiele (privat oder an einem öffentlichen Ort) hat keinen signifikanten Einfluss mehr auf nationalistische Einstellungen, sobald das Erleben von Emotionen und die Aneignung nationaler Symbole berücksichtigt werden. Für die neu aufgenommenen Variablen lassen sich die erwarteten positiven Effekte erkennen: Unterstellt man, dass sich nationalistische Einstellungen von 
t1 zu t2 bei jenen Befragten, die keine positiven Emotionen beim Ansehen der EM-Spiele erlebten, nicht verändert haben, so lässt sich für Befragte mit sehr starken positiven Emotionen ein Zuwachs im Nationalismus von 0,57 Skalenpunkten schätzen. Der Effekt für die Aneignung nationaler Symbole ist von ähnlicher Stärke: Hier lässt sich ein Zuwachs von 0,54 Punkten auf der Nationalismus-Skala erwarten, wenn die Befragten mehr als drei Handlungen angaben, durch die sie sich im Verlauf der Fußball-EM nationale Symbole angeeignet haben. Da bei den Befragten, die sowohl von starken Emotionen als auch von der Aneignung nationaler Symbole berichten, beide Effekte addiert werden müssen, kann das Nationalismus-Niveau durch die EM-Spiele systematisch um mehr als einen Skalenpunkt beeinflusst werden.

Für das komplexeste Modell (IV) wird eine Modellgüte $\left(\mathrm{R}^{2}\right)$ von 0,42 ausgewiesen. Ein Großteil der Erklärungsleistung geht aber auch hier auf die vor der EM erhobenen Nationalismus-Werte zurück, die gleich im ersten Modellierungsschritt kontrolliert werden. Erst aus der Differenz der Modellgüte zwischen Modell I und Modell IV lässt sich ablesen, wie gut sich die nationalistischen Einstellungen durch die Fußball-EM erklären lassen: Der Zuwachs im $\mathrm{R}^{2}$ von 0,10 zeigt, dass sich die Nationalismus-Werte zum Zeitpunkt t 2 zu insgesamt $10 \%$ auf die Fußball-EM zurückführen lassen.

\subsection{Patriotismus und Nationalismus nach der Fußball-EM 2012}

Hält der Nationalstolz der Befragten auch nach Ende der Europameisterschaft noch an? Um diese Frage zu prüfen, werden in den entsprechenden Modellen nun die Patriotismus- bzw. Nationalismus-Werte aus der dritten Befragung, zwei Wochen nach dem Ausscheiden der DFB-Auswahl, eingesetzt ( $\mathrm{t} 3$ ). Die Regressionsmodelle prüfen also, ob es eine Veränderung von $\mathrm{t} 1 \mathrm{zu}$ t3 gegeben hat, die sich systematisch auf die Fußball-EM zurückführen lässt (vgl. Tab.4).

(1) In den Regressionsanalysen zur Veränderung des Patriotismus (P-I bis P-III) werden noch immer signifikante Effekte ausgewiesen: Das Ansehen der EM-Spiele der deutschen Mannschaft beeinflusst auch zwei Wochen nach dem Ausscheiden des DFB-Teams noch das Patriotismus-Niveau der Befragten positiv. Im zweiten Modell (P-II) fällt der Effekt etwas schwächer aus als zur Befragung während der EM (0,34 statt 0,37). Berücksichtigt man zusätzlich, wie viele Spiele die Befragten beim Public Viewing gesehen

Tab. 4: Patriotismus und Nationalismus nach Ende der Fußball-EM 2012

\begin{tabular}{llllllll}
\hline & \multicolumn{2}{l}{$\begin{array}{l}\text { Patriotismus } \\
\text { nach der EM (t3) }\end{array}$} & \multicolumn{4}{l}{$\begin{array}{l}\text { Nationalismus } \\
\text { nach der EM (t3) }\end{array}$} \\
\cline { 2 - 8 } & P-I & P-II & P-III & N-I & N-II & N-III \\
\hline Patriotismus/Nationalismus vor EM (t1) & $0,82^{*}$ & $0,76^{*}$ & $0,75^{*}$ & $0,60^{*}$ & $0,57^{*}$ & $0,57^{*}$ \\
angesehene Spiele (DFB-Auswahl) & - & $0,34^{*}$ & 0,20 & - & $0,17^{+}$ & 0,13 \\
Public Viewing (Spiele DFB-Auswahl) & - & - & $0,17^{*}$ & - & - & 0,05 \\
Modellgüte ( $\left.\mathrm{R}^{2}\right)$ & 0,57 & 0,61 & 0,63 & 0,43 & 0,45 & 0,45 \\
\hline
\end{tabular}

Studierendenbefragung. Lineare Regressionsanalysen. Angegeben sind unstandardisierte Regressionskoeffizienten (b)

${ }^{*} p<0,05 ;{ }^{+} p<0,10$ (einseitiger Signifikanztest) 
haben, zeigt sich ein markanter Unterschied: Zwei Wochen nach dem Aus für die deutsche Mannschaft stimmen die Befragten, die die EM-Spiele an einem öffentlichen Ort gesehen haben, den patriotischen Aussagen noch stärker zu als jene Befragten, die sich die Spiele zu Hause angesehen haben. Public Viewing scheint also, zumindest im Hinblick auf patriotische Einstellungen, einen stärkeren Nachhall zu haben, der auch kurz nach der EM noch zu beobachten ist.

(2) Die Analysen zum Nationalismus (N-I bis N-III) zeigen ein etwas anderes Bild: Zwei Wochen nach dem Turnier-Aus der deutschen Nationalmannschaft sind nur noch schwache Effekte zu erkennen. Das zweite Modell (N-II) zeigt zwar noch immer einen positiven Effekt für das Ansehen der EM-Spiele auf nationalistische Einstellungen an, der auch auf dem 0,10-Niveau signifikant ausfällt. Im Vergleich zu den Zusammenhängen während der EM ist der Effekt nach der EM aber schon erkennbar schwächer geworden $(0,17$ statt 0,22). Für die Teilnahme am Public Viewing ist nach Ende der EM ebenfalls kein substanzieller Einfluss mehr auf nationalistische Einstellungen zu erkennen. Der leichte Anstieg des Nationalismus während der EM war kurze Zeit später bereits am Abklingen.

Zwei Wochen nach dem verlorenen Halbfinale und neun Tage nach dem Ende der EM lässt sich ein Rückgang der Patriotismus- und Nationalismus-Werte auf das Ausgangsniveau vier Wochen vor dem Turnier erst ansatzweise an den Daten nachvollziehen. Die Effekte der EM werden schwächer - bei nationalistischen Einstellungen scheint das eher und schneller der Fall zu sein als bei patriotischen Einstellungen. Es ist jedoch nicht anzunehmen, dass die EM dauerhaft zu einem größeren Patriotismus oder Nationalismus bei den Befragten geführt hat. Vielmehr ist zu vermuten, dass der dritte Befragungszeitpunkt noch zu dicht an der Fußball-EM lag. Mit einem größeren zeitlichen Abstand zum Turnier dürften die nationalen Zugehörigkeitsgefühle ihre EM-bedingte hohe Salienz immer mehr verlieren, sodass sich die Patriotismus- und Nationalismus-Werte schließlich wieder auf dem Niveau vor der EM einpendeln müssten. Zu belegen ist dies mit den hier analysierten Daten allerdings nicht. ${ }^{9}$

\section{Resümee}

Die eingangs aufgeworfenen Fragen können auf Basis der empirischen Befunde nun etwas genauer beantwortet werden: Zunächst legen die Ergebnisse nahe, dass durch die Rezeption der Fußball-EM die Identifikation der Befragten mit dem Nationalstaat zugenommen hat. Bereits das Ansehen der Spiele der deutschen Mannschaft in der privaten Wohnung ist mit einer stärkeren nationalen Identifikation (Patriotismus, Nationalismus) verbunden. Noch stärker ist der Zuwachs an nationaler Identifikation, wenn die Spiele in der Öffentlichkeit angesehen werden. Beim Public Viewing steigert sich zum einen die emotionale Erregung durch die Anwesenheit Gleichgesinnter, zum anderen ist das Fuß-

9 Eine Befragung der Studierenden in einem größeren zeitlichen Abstand zur EM war innerhalb der Vorlesungszeit des Sommersemesters nicht zu realisieren. Eine weitere Befragung in die Semesterferien zu legen, erschien ebenfalls wenig Erfolg versprechend, da geringe Rücklaufquoten zu erwarten gewesen wären, die wiederum die Fallzahlen gemindert und eine im Längsschnitt angelegte Analyse unmöglich gemacht hätten. 
ballspiel dort stärker eingerahmt in nationale Symbole, die sich die Anwesenden aktiv aneignen. Beide Einflussfaktoren - starke positive Emotionen und Aneignung nationaler Symbole - sind, wie die Befunde zeigen, für die stärkere nationale Identifikation der Befragten verantwortlich.

Die Befunde geben darüber hinaus Anhaltspunkte dafür, welche Art von nationaler Identifikation die Fußball-EM generiert hat: In den gesellschaftspolitischen Debatten dominiert weitestgehend eine Sichtweise, die den Nationalstolz, der auf den Fanmeilen zum Ausdruck kommt, als „Party-Patriotismus“ oder „Patriotismus light“ beschreibt, bei dem das gemeinsame Feiern im Vordergrund steht und der sich nicht gegen Fremde oder andere Nationen richtet. Die Befunde zeigen, dass Aussagen, die eine patriotische, emotionale Verbundenheit zur Nation ausdrücken, während der EM besonders starke Zustimmung erhalten. Allerdings gibt es auch einen systematischen Effekt auf nationalistische Einstellungen: Aussagen, die auf die Überlegenheit der eigenen Nation und die unbedingte, kritiklose Treue zum Nationalstaat abheben, erhalten während der EM ebenfalls mehr Zustimmung. Die Zusammenhänge sind hier zwar schwächer, aber ebenfalls signifikant.

Wie schon in der Studie von Hilde Weiss und Christoph Reinprecht (1998) deutlich wird, sind Nationalismus und Patriotismus nach dem hier favorisierten Verständnis benachbarte und keinesfalls einander entgegengesetzte Konzepte. Zwischen der im Beitrag verwendeten Patriotismus- und Nationalismus-Skala besteht eine positive Korrelation $(r=0,48)$. Allein daraus lässt sich schlussfolgern, dass es kaum ein Ereignis geben dürfte, dass sich nur auf eines der beiden Konstrukte auswirkt, nicht aber auf das andere. Im Schlepptau des „Party-Patriotismus“ auf den Public Viewing-Plätzen und Fanmeilen kommt auch eine Portion nationale Überheblichkeit und Abwertung der anderen daher. Nachhaltigen Einfluss dürften diese Effekte der Fußball-EM jedoch nicht ausüben, denn bereits kurz nach dem Ende des Turniers schwächen sie sich merklich ab. Sobald die Emotionen verblassen und die Fahnen abmontiert sind, rückt die Nation wieder in den Hintergrund. Junge (2008) sieht Public Viewing als prototypisches Beispiel für flüchtige, episodenhafte Formen der Vergemeinschaftung: Die kollektive Einheit zerfällt in dem Moment, in dem sich der Erregungszustand auflöst, die Selbstreflexion einsetzt und die individuellen Unterschiede im Selbsterleben und die damit verbundenen Abgrenzungen zu den anderen an Bedeutung gewinnen. Was dann bleibt, ist ihm zufolge die Sehnsucht nach dem nächsten (Sport-)Event, bei dem man abermals kurzzeitig Gemeinschaft und kollektive Gefühle erleben kann.

Eine methodische Einschränkung der vorliegenden Studie ergibt sich ohne Zweifel aus der Stichprobe, die ausnahmslos aus Studierenden der Sportwissenschaften besteht. Die befragten Studierenden dürften sich insbesondere durch zwei Eigenschaften von der Durchschnittsbevölkerung abheben: Zum einen kann man ihnen ein ausgeprägtes Interesse für Sport unterstellen und folglich vermuten, dass sie die Fußball-EM mit großer Aufmerksamkeit verfolgt haben. Von den hier befragten Studierenden hat die Mehrheit alle EM-Spiele des DFB-Teams angesehen, sodass ihre nationale Identifikation zumindest potenziell auch beeinflusst werden konnte. Wer die EM-Spiele aus Mangel an Fußballinteresse nicht rezipiert, der kann durch die EM auch keinen Nationalstolz entwickeln. Zum anderen handelt es sich bei den Befragten um eine jüngere Altersgruppe. Diese Eigenschaft dürfte für die Studie ebenfalls von Relevanz sein, denn jüngere Befragte, die den 
Zweiten Weltkrieg und den NS-Terror nur aus dem Geschichtsunterricht kennen, dürften ein weitaus ,unverkrampfteres“ Verhältnis zum Nationalstaat und zu nationalen Symbolen haben als noch die Generationen ihrer Eltern und Großeltern. Beide Eigenschaften der Stichprobe - ausgeprägtes Fußballinteresse und jüngeres Alter - können deshalb dazu führen, dass hier die Zusammenhänge vergleichsweise stark ausfallen. Womöglich wären die Effekte der Fußball-EM auf nationale Identifikation in einer repräsentativen Bevölkerungsstichprobe etwas schwächer. Dass sich dort keine oder gänzlich andere Zusammenhänge zeigen würden als bei den befragten Sportstudierenden, ist allerdings nicht wahrscheinlich.

Danksagung: Mein großer Dank gebührt Ulrike Burrmann, Miriam Seyda (Technische Universität Dortmund) und Tina Nobis (Humboldt-Universität zu Berlin), die einen Teil der Daten, auf denen dieser Beitrag basiert, erhoben haben. Ohne ihre Kooperation und ihr Engagement wäre eine im Längsschnitt angelegte Stichprobe nicht zu realisieren gewesen.

Open Access: Dieser Artikel unterliegt den Bedingungen der Creative Commons Attribution License. Dadurch sind die Nutzung, Verteilung und Reproduktion erlaubt, sofern der/die Originalautor/en und die Quelle angegeben sind.

\section{Literatur}

Adorno, T. W., Frenkel-Brunswik, E., Levinson, D. J., \& Sanford, R. N. (1950). The authoritarian personality. New York: Harper \& Brothers.

Ahlheim, K., \& Heger, B. (2008). Nation und Exklusion: Der Stolz der Deutschen und seine Nebenwirkungen. Schwalbach: Wochenschau Verlag.

Anderson, B. (1983). Immagined communities. Reflections on the origin and spread of nationalism. London: Verso.

Ashmore, R. D., Deaux, K., \& McLaughlin-Volpe, T. (2004). An organizing framework for collective identity: Articulation and significance of multidimensionality. Psychological Bulletin, $130,80-114$.

Brewer, M. B. (1991). The social self: On being the same and different at the same time. Personality and Social Psychology Bulletin, 17, 475-482.

Brubaker, R., \& Cooper, F. (2000). Beyond „identity“. Theory and Society, 29, 1-47.

Calhoun, C. (Hrsg.). (1994). Social theory and the politics of identity. Cambridge: Blackwell.

Calhoun, C. (2005). Constitutional patriotism and the public sphere: Interests, identity, and solidarity in the integration of Europe. International Journal of Politics, Culture, and Society, 18, 257-280.

Cottingham, M. D. (2012). Interaction ritual theory and sports fans: Emotions, symbols, and solidarity. Sociology of Sport Journal, 29, 168-185.

Delhey, J. (2004). Nationales und transnationales Vertrauen in der Europäischen Union. Leviathan, $32,15-45$.

Dembowski, G. (2009). Wie weich ist Nationalismus im deutschen Fußball? In Projektgruppe Nationalismuskritik (Hrsg.), Irrsinn der Normalität. Aspekte der Reartikulation des deutschen Nationalismus (S. 182-205). Münster: Westfälisches Dampfboot.

Depenheuer, O. (1995). Integration durch Verfassung? Zum Identitätskonzept des Verfassungspatriotismus. German American Law Journal, 5, 95-114.

Durkheim, E. (1981). Die elementaren Formen des religiösen Lebens. Frankfurt a. M.: Suhrkamp. 
Eisenstadt, S. N., \& Giesen, B. (1995). The construction of collective identity. European Journal of Sociology, 36, 72-102.

Fleiß, J., Höllinger, F., \& Kuzmics, H. (2009). Nationalstolz zwischen Patriotismus und Nationalismus? Empirisch-methodologische Analysen und Reflexionen am Beispiel des ISSP 2003 „National Identity“. Berliner Journal für Soziologie, 19, 409-434.

Fuchs, D., Gerhards, J., \& Roller, E. (1993). Wir und die Anderen. „Imagined Communities“ im westeuropäischen Vergleich. WZB Discussion Paper FS III 93-301. Berlin: WZB.

Gebauer, G. (1996). Der neue Nationalismus im Sport. In G. Gebauer (Hrsg.), Olympische Spiele - die andere Utopie der Moderne (S. 264-269). Frankfurt a. M.: Suhrkamp.

Hebeker, E., \& Hildmann, P. W. (Hrsg.). (2007). Fröhlicher Patriotismus? Eine WM-Nachlese. München: Hanns-Seidel-Stiftung.

Hobsbawm, E. (1991). Nationen und Nationalismus: Mythos und Realität seit 1780. Frankfurt a. M.: Campus.

Houlihan, B. (1997). Sport, national identity, and public policy. Nations and Nationalism, 3, $113-137$.

Hroch, M. (2005). Das Europa der Nationen: Die moderne Nationsbildung im europäischen Vergleich. Göttingen: Vandenhoeck \& Ruprecht.

Ismer, S. (2011). Embodying the nation: Football, emotions, and the construction of collective identity. Nationalities Papers, 39, 547-565.

Jenkins, R. (1996). Social identity. London: Routledge.

Junge, M. (2008). Die kollektive Erregung des public viewing - oder: die Tragödie der Identifikation und der Sozialität. In R. Hitzler, A. Honer, \& M. Pfadenhauer (Hrsg.), Posttraditionale Gemeinschaften (S. 189-201). Wiesbaden: VS Verlag für Sozialwissenschaften.

Kaina, V. (2009). Wir in Europa. Kollektive Identität und Demokratie in der Europäischen Union. Wiesbaden: VS Verlag für Sozialwissenschaften.

Karolewski, I. P., \& Suszycki, A. M. (2011). The nation and nationalism in Europe. Edinburgh: Edinburgh University Press.

Kosterman, R., \& Feshbach, S. (1989). Toward a measure of patriotic and nationalistic attitudes. Political Psychology, 10, 257-274.

Lepsius, M. R. (1982). Nation und Nationalismus in Deutschland. In H. A. Winkler (Hrsg.), Nationalismus in der Welt von heute. Geschichte und Gesellschaft, Sonderheft 8 (S. 12-27). Göttingen: Vandenhoeck \& Ruprecht.

Levinson, D. J. (1950). The study of ethnocentric ideology. In T. W. Adorno, E. Frenkel-Brunswik, D. J. Levinson, \& R. N. Sanford, The authoritarian personality (S. 102-150). New York: Harper \& Brothers.

Luhtanen, R., \& Crocker, J. (1992). A collective self-esteem scale: Self-evaluation of one's social identity. Personality and Social Psychology Bulletin, 18, 302-318.

Riedl, L. (2008). „Und dann jubelte das ganze Stadion!“ Zur Entstehung und Steuerung kollektiver Emotionen im Spitzensport. Sport und Gesellschaft, 5, 221-250.

Rorty, R. (1998). Achieving our country. Leftist thought in twentieth-century America. Cambridge: Harvard University Press.

Schäfer, M. S. (2010). Fans und Emotionen. In J. Roose, M. S. Schäfer, \& T. Schmidt-Lux (Hrsg.), Fans. Soziologische Perspektiven (S.109-132). Wiesbaden: VS Verlag für Sozialwissenschaften.

Schediwy, D. (2012). Ganz entspannt in Schwarz-Rot-Gold? Der neue deutsche Fußballpatriotismus aus sozialpsychologischer Perspektive. Münster: LIT.

Schrag, D. (2009). „Flagging the nation“ in international sport: A Chinese olympics and a German world cup. The International Journal of the History of Sport, 26, 1084-1104.

Sen, A. (2007). Die Identitätsfalle. München: Beck.

Tajfel, H. (Hrsg.). (1982). Social identity and intergroup relations. Cambridge: Cambridge University Press. 
Tajfel, H., \& Turner, J. C. (1986). The social identity theory of intergroup behavior. In S. Worchel \& W. Austin (Hrsg.), Psychology of intergroup relations (S. 7-24). Chicago: Nelson-Hall.

Turner, J. C. (1982). Towards a cognitive redefinition of the social group. In H. Tajfel (Hrsg.), Social identity and intergroup relations (S. 15-40). Cambridge: Cambridge University Press.

Turner, J. C., Hogg, M. A., Oakes, P. J., Reicher, S. D., \& Wetherell, M. S. (1987). Rediscovering the social group. A self-categorization theory. New York: Basil Blackwell.

van Hilvoorde, I., Elling, A., \& Stokvis, R. (2010). How to influence national pride? The olympic medal index as a unifying narrative. International Review for the Sociology of Sport, 45, 87-102.

von Scheve, C., Beyer, M., Ismer, S., Kozlowska, M., \& Morawetz, C. (2012). Collective emotions and national identification: A natural experiment around the Football World Cup. Unveröffentlichtes Manuskript, Freie Universität Berlin.

Weiss, H., Donat, E., \& Latcheva, R. (2009a). Patriotismus-Items der Wiener „Patriotismus- und Nationalismus-Skala“. In A. Glöckner-Rist (Hrsg.), Zusammenstellung sozialwissenschaftlicher Items und Skalen. ZIS Version 13.00. Bonn: GESIS.

Weiss, H., Donat, E., \& Latcheva, R. (2009b). Nationalismus-Items der Wiener „Patriotismus- und Nationalismus-Skala“. In A. Glöckner-Rist (Hrsg.), Zusammenstellung sozialwissenschaftlicher Items und Skalen. ZIS Version 13.00. Bonn: GESIS.

Weiss, H., \& Reinprecht, C. (1998). Demokratischer Patriotismus oder ethnischer Nationalismus in Ost-Mitteleuropa? Empirische Analysen zur nationalen Identität in Ungarn, Tschechien, Slowakei und Polen. Wien: Böhlau.

Wieviorka, M. (2003). Kulturelle Differenzen und kollektive Identitäten. Hamburg: Hamburger Edition.

Michael Mutz, geb. 1980. Juniorprofessor für Sportsoziologie an der Georg-August-Universität Göttingen. Arbeitsschwerpunkte: Sportsoziologie, Sozialisationsforschung, Jugendsoziologie, empirische Sozialforschung. Ausgewählte Veröffentlichungen: Sport als Sprungbrett in die Gesellschaft, 2012; Athletic participation and the approval and use of violence, in: European Journal for Sport and Society, 2012; (mit N. Schmiade) Sportliche Eltern, sportliche Kinder - Die Sportbeteiligung von Vorschulkindern im Kontext sozialer Ungleichheit, in: Sportwissenschaft, 2012. 